\title{
MMMVI: Detecting SARS-CoV-2 Variants of Concern in Metagenomic Wastewater Samples
}

\author{
Dillon O.R. Barker ${ }^{1, \dagger, *}$, Cody J. Buchanan ${ }^{1, \dagger, *}$, Chrystal Landgraff ${ }^{1,2}$, \\ Eduardo N. Taboada ${ }^{1}$
}

${ }^{1}$ Division of Enteric Diseases, National Microbiology Laboratory, Public Health Agency of Canada, 1015 Arlington Street, R3E 3R2, Winnipeg, Manitoba, Canada

${ }^{2}$ Food Science Department, University of Guelph, 50 Stone Road East, N1G 2W1, Guelph, Ontario, Canada

${ }^{\dagger}$ Authors contributed equally to the work.

*To whom correspondence should be addressed.

\begin{abstract}
Motivation: SARS-CoV-2 is the causative agent of the COVID-19 pandemic. Variants of Concern (VOCs) and Variants of Interest (VOIs) are lineages that represent a greater risk to public health, and can be differentiated from the wildtype virus based on unique profiles of signature mutations. Metagenomic sequence analysis of wastewater represents an emerging form of surveillance that can capture early signal for these variants in a community prior to detection through public health testing or genomic surveillance activities. However, because multiple viral genomes are likely to be present in a metagenomic sample, additional analytical scrutiny of the sequencing reads beyond variant calling is needed to increase confidence in diagnostic determinations.

Results: Where multiple signature mutations are present on a given read, they can be used as enhanced biomarkers to confirm the presence of a VOC/VOI in the sample. We have developed MMMVI, a tool to aggregate and report on the likely presence of a VOC/VOI in a set of metagenomic reads based on the detection of reads bearing multiple signature mutations.

Availability: MMMVI is implemented in Python, and is available under the MIT licence from https://github.com/dorbarker/voc-identify/

Contact: dillon.barker@canada.ca
\end{abstract}

\section{Introduction}

Wastewater testing for SARS-CoV-2 can offer several advantages that can help address testing gaps and complement more traditional forms of laboratory-based surveillance. Wastewater surveillance is non-invasive, can capture signal from asymptomatic and positive cases not detected via the public health system, and can detect early-warning data predictive of future clinical trends (Medema et al., 2020; D'Aoust et al., 2021). Metagenomic sequencing provides a mechanism to characterize the genetic diversity of SARS-CoV-2 present in wastewater, and presents a snapshot of the lineages circulating within the community. Importantly, these data can be screened for the presence of SARS-CoV-2 Variants of Concern (VOCs) and Variants of Interest (VOIs) based on the profile of signature mutations observed in the sample (Hadfield et al., 2018; Rambaut et al., 2020; Public Health England, 2021). However, a sample may not contain the complete set of mutations corresponding to any single VOC/VOI. This could be due to incomplete sequencing of the genome, or indicative of the early introduction, or localized outbreak of one or more VOCs/VOIs in the community. It is also possible to observe signature mutations from multiple VOCs/VOIs in a single sample, and some signature mutations (e.g., $\mathrm{N} 501 \mathrm{Y})$ have emerged independently in multiple VOCs/VOIs. Thus, the criteria (i.e., the number and/or set of specific signature mutations required) to stipulate the presence of a VOC/VOI in a metagenomics context are not well defined.

We propose an approach that takes advantage of signature mutation that occur in close proximity to one another in the SARS-CoV-2 genome. If we assume that an individual sequencing read is ultimately derived from a distinct viral genome, then any mutations identified on that read should also be derived from that viral genome. Therefore, the combination of signature mutations found on a single read can be used as an enhanced biomarker that is more sensitive than variant calling for the detection of a VOC/VOI, but also suitable as a surrogate marker in samples where the genome is incompletely sequenced.

Here, we present MMMVI, a command line tool that automates the categorisation and quantification of reads based on all combination of signature mutations they encompass. It generates a number of detailed reports to more confidently inform the presence of, or differentiate between, one or more VOCs/VOIs in a sample. MMMVI is compatible with data generated from both Illumina and Oxford Nanopore Technologies platforms, and can be readily run both on consumer-grade hardware and in parallel on a computer cluster. 


\section{Description}

\subsection{Data}

A wastewater sample positive by RT-qPCR detection for the B.1.1.7/N501Y.V1/Alpha SARS-CoV-2 VOC was amplified using a tiledPCR amplification method and sequenced on both the Illumina MiSec and Oxford Nanopore Technologies MinION platforms (BioProject: PRJNA708265). Reads from each sequencing run were independently mapped to the reference genome, SARS-CoV-2 isolate Wuhan-Hu-1 (MN908947.3) (Wu et al., 2020).

\subsection{Input}

MMMVI takes three files as input: the sample in BAM format aligned to the reference genome ( $\mathrm{Li}$ et al., 2009), the complete reference genome in FASTA format, and variant definitions. These definitions can be provided either as a tabular text file matching the format described in Table 1, or as YAML files matching the Public Health England Standardised Variant Definitions specification (Public Health England, 2021).

Table 1. Example of the tabular mutation file format required by MMMVI.

\begin{tabular}{lr}
\hline VOC/VOI & Mutation \\
\hline B.1.1.7 & C23604A \\
B.1.1.7 & GAT28280CTA \\
B.1.1.7 & {$[21765-21770]$ del } \\
P.1 & 28262 AACA \\
\hline
\end{tabular}

The first line in Table 1 describes substitution of $\mathrm{C}$ in the wildtype by A in B.1.1.7 at reference genome position 23604 in the sense strand Similarly, the second line shows a three base substitution of GAT by CTA beginning at position 28280 . The third line is a 6 base deletion relative to the reference beginning at position 21765 . On the fourth, a four base insertion between reference positions 28262 and 28263 is indicated. All positions are 1-based, i.e. position 1 is the first base in the reference genome. All intervals are closed.

\subsection{Mutation Identification}

MMMVI makes use of pandas and pysam (https://github.com/pysamdevelopers/pysam) (McKinney et al., 2011; PySAM Developers, 2019) for efficient processing.

Each read is compared to the set of mutations provided by the mutations table. If a read spans all the reference positions for a given mutation, it sequence at those positions is compared with the mutation. If the rea contains all of the substitutions or deletions described by the mutation, the read is considered to have that mutation. Gaps in the read alignment are considered deletions. A read may contain multiple mutations, and each unique combination of mutations is considered a read species.

\subsection{Output}

All outputs are returned to the user as delimited text files, with the delimiter optionally selectable by the user.

\subsubsection{Summary Table}

The summary file contains a table indicating the count of reads possessing $N$ mutations for each VOC/VOI, as well as the wildtype. The numerica column headers indicate $N$ mutations found on the read, and the left column shows the variant names. The table values for these show the coun of reads with $N$ mutations for each variant. The maximum mutation per median read indicates the greatest theoretical number of mutations for a given VOC/VOI that can fit on a single read based on the median read length calculated for the sample. Also shown are the fraction of signature mutations that are represented in the sample, as well the fraction of signature mutations which are exclusive to that VOC/VOI.

Table 2. An example of the summary table produced by MMMVI from Illumina sequencing of a metagenomic wastewater sample.

\begin{tabular}{lrrrrrrr}
\hline VOC/VOI & 0 & 1 & 2 & 3 & 4 & 5 & 6 \\
\hline reference & 1956464 & 596843 & 33351 & 7539 & 15771 & 4396 & 15 \\
B.1.1.7 & 2505105 & 68390 & 10977 & 22169 & 7738 & 0 & 0 \\
P.1 & 2614337 & 42 & 0 & 0 & 0 & 0 & 0 \\
\hline \multicolumn{5}{c}{ Max. } & Complete & Exclusive & \\
& Mutations & $/$ & Signature & Signature & \\
& Median Read & Mutations & Mutations & \\
reference & 6 & $40 / 42$ & $0 / 0$ & \\
B.1.1.7 & 4 & $20 / 24$ & $19 / 21$ & \\
P.1 & 3 & $8 / 21$ & $7 / 18$ &
\end{tabular}

\subsubsection{Co-occurrence Matrices}

The co-occurrence matrices show the frequencies of cases where multiple mutations are present on a given read.

These matrices are given both as absolute counts and as relative counts. The relative count expresses, for each pair of mutations $X$ and $Y$, the count of reads exhibiting both $X$ and $Y$ as a proportion of the count of all reads possessing $X$.

\subsubsection{Read Report}

This report shows every sequencing read given as input and alleles presen for each VOC/VOI and the wildtype. The alleles are expressed as genomic positions, with coordinates placed in the column or columns which correspond to a VOC or the wildtype.

\subsubsection{Read Species Report}

The read species report shows every unique combination of mutations found on a single read, as well as the positions that are mutated, the nucleotide or INDEL present at those positions, and the number of times a given combination was observed in the dataset.

Further, it provides a bit array for each VOC/VOI and the wildtype, indicating at each position which variant shares the observed nucleotide or INDEL. Finally, each combination is expressed as a proportion relative to the number of reads spanning the relevant positions, and as a proportion relative to the total number of reads in the dataset.

\section{Conclusion}

MMMVI uses a command line interface for user interaction. For ease of automation, we also provide a Snakemake workflow for executing MMMVI jobs (Mölder et al., 2021)

Table 3 shows the performance of MMMVI on inputs generated using the Illumina MiSeq and Oxford Nanopore Technologies MinION. We searched for eleven VOCs or VOIs plus wildtype, comprising 153 variant positions.

MMMVI facilitates detection of SARS-CoV-2 VOCs in metagenomic sequence data sourced from wastewater. Although it was designed for use with wastewater samples, we believe this approach is applicable to other sample types. 
Table 3. Computing resource consumption on example inputs when searching for eleven variants and the wildtype.

\begin{tabular}{llll}
\hline Instrument & Read Count & Elapsed (min) & $\begin{array}{l}\text { Memory Usage } \\
(\mathrm{GB})\end{array}$ \\
\hline MiSeq & 2614393 & 44 & 9.32 \\
MinION & 84078 & 8 & 0.48 \\
\hline
\end{tabular}

An example of results generated by MMMVI using Illumina MiSeq and Oxford Nanopore Technologies MinION data from BioProject PRJNA708265 are provided as Supplemental Data.

\section{Acknowledgements}

We would like to thank Robert Delatolla, Tyson Graber and Lawrence Goodridge for organising the collection of and providing access to the wastewater sample used for this analysis.

\section{References}

D’Aoust, P. M., Graber, T. E., Mercier, E., Montpetit, D., Alexandrov, I., Neault, N., Baig, A. T., Mayne, J., Zhang, X., Alain, T., et al. (2021). Catching a resurgence: Increase in sars-cov-2 viral rna identified in wastewater $48 \mathrm{~h}$ before covid-19 clinical tests and $96 \mathrm{~h}$ before hospitalizations. Science of The Total Environment, 770, 145319.

Hadfield, J., Megill, C., Bell, S. M., Huddleston, J., Potter, B., Callender,

C., Sagulenko, P., Bedford, T., and Neher, R. A. (2018). Nextstrain: real-time tracking of pathogen evolution. Bioinformatics, 34(23), 41214123

Li, H., Handsaker, B., Wysoker, A., Fennell, T., Ruan, J., Homer, N., Marth, G., Abecasis, G., Durbin, R., and Subgroup, . G. P. D. P. (2009). The Sequence Alignment/Map format and SAMtools. Bioinformatics, 25(16), 2078-2079.

McKinney, W. et al. (2011). pandas: a foundational Python library for data analysis and statistics. Python for High Performance and Scientific Computing, 14(9), 1-9.

Medema, G., Been, F., Heijnen, L., and Petterson, S. (2020). Implementation of environmental surveillance for sars-cov-2 virus to support public health decisions: Opportunities and challenges. Current Opinion in Environmental Science \& Health, 17, 49-71.

Mölder, F., Jablonski, K. P., Letcher, B., Hall, M. B., Tomkins-Tinch, C. H., Sochat, V., Forster, J., Lee, S., Twardziok, S. O., Kanitz, A., et al. (2021). Sustainable data analysis with Snakemake. F1000Research, 10(33), 33.

Public Health England (2021). Public Health England: Standardised Variant Definitions.

PySAM Developers (2019). pysam: htslib interface for Python

Rambaut, A., Holmes, E. C., O’Toole, Á., Hill, V., McCrone, J. T., Ruis, C., du Plessis, L., and Pybus, O. G. (2020). A dynamic nomenclature proposal for SARS-CoV-2 lineages to assist genomic epidemiology. Nature Microbiology, 5(11), 1403-1407.

Wu, F., Zhao, S., Yu, B., Chen, Y.-M., Wang, W., Song, Z.-G., Hu, Y., Tao, Z.-W., Tian, J.-H., Pei, Y.-Y., et al. (2020). A new coronavirus associated with human respiratory disease in China. Nature, 579(7798), 265-269. 